\title{
Germanica
}

\section{L'écriture de la guerre en RDA dans les années cinquante}

L'exemple de Franz Fühmann

Schreiben über den Krieg in der DDR der 1950er Jahre am Beispiel Franz

Fühmanns

\section{Carola Hähnel-Mesnard}

\section{CpenEdition}

Journals

Édition électronique

URL : http://journals.openedition.org/germanica/518

DOI : 10.4000/germanica.518

ISSN : 2107-0784

Éditeur

Université de Lille

\section{Édition imprimée}

Date de publication : 1 juin 2008

Pagination : 89-118

ISBN : 978-2-913857-21-6

ISSN : 0984-2632

\section{Référence électronique}

Carola Hähnel-Mesnard, «L'écriture de la guerre en RDA dans les années cinquante », Germanica [En

ligne], 42 | 2008, mis en ligne le 01 juin 2010, consulté le 06 octobre 2020. URL : http://

journals.openedition.org/germanica/518; DOI : https://doi.org/10.4000/germanica.518

Ce document a été généré automatiquement le 6 octobre 2020.

(c) Tous droits réservés 


\title{
L'écriture de la guerre en RDA dans les années cinquante
}

\author{
L'exemple de Franz Fühmann \\ Schreiben über den Krieg in der DDR der 1950er Jahre am Beispiel Franz \\ Fühmanns
}

Carola Hähnel-Mesnard

1 Dans Vingt-deux jours ou La moitié de la vie, journal d'un voyage à Budapest, Franz Fühmann revient de façon insistante sur le sujet qui a travaillé toute son œuvre de l'intérieur et qui le touche au plus profond de lui-même : la conversion (Wandlung) à l'antifascisme d'un soldat entièrement dévoué au national-socialisme. Fühmann évoque le bouleversement provoqué par ce qu'il nomme la «nouvelle de Nuremberg et d'Auschwitz ${ }^{1} »$ qui l'avait mis soudainement devant ses responsabilités, alors qu'il était détenu dans un camp de rééducation soviétique où il est resté pendant cinq ans. À partir de ce moment, il lui a fallu se poser la question : que savait-il d'Auschwitz et comment aurait-il agi s'il y avait été en poste. À cela, Fühmann répond qu'il y aurait fait "son devoir ». Il va même jusqu'à reconnaître sa responsabilité dans le fait d'avoir « permis à Auschwitz d'exister » en exerçant ses fonctions pendant la guerre de façon exemplaire (Vdj, 221).

Dans un autre texte, Fühmann est plus explicite encore. Alors qu'il affirme ne pas avoir commis de crime (simplement parce qu'on ne lui en a pas donné l'ordre) et d'avoir eu, au sortir de la guerre, la "conscience d'une non-culpabilité individuelle ${ }^{2}$ ", il est profondément tourmenté par l'idée d'être co-responsable d'Auschwitz:

L'expérience bouleversante qui, en fin de compte, a changé ma vie, était désormais que moi, alors que d'un point de vue individuel je me considérais comme noncoupable, j'avais néanmoins protégé Auschwitz. Il ne s'agissait pas d'un autre qui, dans un lointain village polonais, letton, français, etc., [...], avait commis des atrocités [...], mais de moi et de mes semblables sans qui Auschwitz n'aurait pas été possible. J'étais une partie de la totalité nationale-socialiste qui fonctionnait exactement comme elle devait fonctionner, et ainsi la différence entre Höß et moi n'était que graduelle; juridique, mais non pas morale et existentielle ${ }^{3}$. 
Cette prise de conscience qu'une non-culpabilité individuelle n'empêche pas une responsabilité collective dans la machine exterminatrice nationale-socialiste est redoublée par la conscience que ce n'est que par le hasard des circonstances qu'il n'a lui-même pas été coupable de crimes. C'est à cela qu'il doit moralement se confronter. Ainsi, il note dans son Journal : «Comment pourrai-je jamais dire que j'ai tiré un trait sur mon passé, alors que je place le hasard qui l'a gouverné avec clémence au rang d'arbitre suprême au-dessus de moi? Tirer un trait sur le passé, cela veut dire s'interroger sur toutes les possibilités et donc aussi sur la plus extrême. » (Vdj, 223)

Ce processus de confrontation au passé entamé en tant que prisonnier de guerre soviétique et qui faisait partie de ce que Fühmann appelait sa « conversion », le conduit à un engagement sans faille auprès du nouvel État est-allemand qui représentait à ses yeux «l'Autre d'Auschwitz» (Vdj, 225), c'est-à-dire le système qui pourrait empêcher qu'Auschwitz se répète. "Ma génération est venue au socialisme en passant par Auschwitz. Toute méditation sur notre conversion doit commencer aux portes de la chambre à gaz, là et pas ailleurs " ( $V d j, 171)$, écrit l'auteur avec une formule désormais connue. Toutefois, on tient moins souvent compte de la nuance qu'il ajoute à cette déclaration à la fin de son journal, nuance dans laquelle Fühmann introduit une dimension critique vis-à-vis de son engagement en RDA. Ainsi, «l'accomplissement de [s]a conversion " avait consisté à se mettre au service de la nouvelle société comme, souligne-t-il, un "outil à la volonté éteinte " $(V d j, 225)$ qui n'avait fait qu'accomplir sans réflexion critique ce qu'on lui avait demandé, renonçant par là-même à transmettre ses propres expériences du passé et la leçon de culpabilité qu'il en avait tirée. Fühmann évoque ici, au début des années soixante-dix, un déni de soi qui fut à l'origine de son engagement pour la nouvelle société. Il est en ce sens exemplaire de ce que Wolfgang Emmerich appelle l'«auto-attachement» au nouvel État héritier de l'antifascisme qui donnait à cette génération la possibilité d'une réparation imaginaire (imaginative Wiedergutmachung ). $^{4}$

5 Si ses premières œuvres au service de la RDA, essentiellement des poèmes panégyriques, témoignent effectivement d'une dénégation de ses propres expériences de guerre, Fühmann revient assez rapidement sur ce sujet. D'abord, en 1953, avec son long poème Die Fahrt nach Stalingrad. Eine Dichtung qui subordonne encore les expériences individuelles de la guerre et l'auto-accusation à l'éloge du nouvel ordre socialiste et de l'Union soviétique. Ensuite, à partir de 1955, avec trois nouvelles écrites strictement dans la perspective de soldats allemands: Kameraden (1955), Das Gottesgericht et Kapitulation (1957). Les deux dernières font partie du recueil Stürzende Schatten (1959) qui comprend également trois récits très courts regroupés sous le titre Das Erinnern, récits qui ne sont plus écrits dans la perspective immédiate de l'expérience de la guerre, mais dans celle de la remémoration de la période du nationalsocialisme. Avant d'analyser de plus près ces nouvelles ainsi que le passage à une démarche "mémorielle ", il est important de situer ces œuvres dans le double contexte littéraire et social de la RDA des années cinquante. 


\section{Les discours sur le passé et la guerre dans les années cinquante en RDA}

6 Si l'on s'en tient à la politique culturelle mise en place en zone d'occupation soviétique et dans la jeune RDA ainsi qu'aux injonctions faites aux écrivains, la confrontation au passé national-socialiste n'était pas à l'ordre du jour. Car dès 1948, en appliquant le premier plan économique biennal dans le domaine de la culture, on réclamait de la part des écrivains qu'ils détournent leur regard du passé et qu'ils représentent la nouvelle société, les bouleversements sociaux à la campagne et les grands chantiers de la (re)construction industrielle. Ainsi, Ulbricht avait critiqué en octobre 1948 le peu de soutien apporté à la réalisation de la réforme agraire: "Pourquoi cette littérature n'existe-t-elle pas? La matière est pourtant là. Mais lorsque le combat fut mené, nous étions seuls. Pendant ce temps-là, nos camarades écrivains ont écrit des romans sur l'exil $[. . .]^{5}$.» Mis à part la fonction identificatrice de l'antifascisme et les rituels mémoriels qui s'élaborent autour, la société de la jeune RDA était résolument tournée vers l'avenir.

7 Toutefois, des récits de guerre furent publiés dès la fin des années quarante. Il s'agissait principalement de textes écrits par une génération abusée - c'est ainsi du moins qu'elle se voyait - qui mettait en avant son vécu et le désarroi provoqué par la guerre. C'était des « textes authentiques d'une littérature des ruines et d'une littérature du Kahlschlag, écrits dans l'intention d'y joindre une confession ${ }^{6} »$. Cette littérature à fort caractère autobiographique était écrite dans un esprit quasi-documentaire qui visait à décrire les souffrances des soldats allemands avec le projet de "reconstruire les biographies ${ }^{7}$ ", contrairement au projet d'écriture de Franz Fühmann. Il n'y avait dans ces textes ni prise de conscience d'une quelconque participation à une entreprise meurtrière ni sentiment de culpabilité. Ils provoquaient toutefois de fortes réactions contre la guerre, et ce pacifisme a vite perdu sa raison d'être dans le contexte de la guerre froide.

$8 \mathrm{Si}$, comme le note Ludwig Renn en 1956, c'est l'héritage militaire de l'époque nazie qui fait obstacle à la création d'une littérature sur la guerre en $\mathrm{RDA}^{8}$, pourtant une nécessité de plus en plus grande de revenir sur cette période se fait sentir. La génération concernée continue à écrire des textes dans le but de s'approprier ses expériences du passé. Tel est le cas de Franz Fühmann qui, depuis la fin de la guerre, essaie d'exprimer son vécu de cette façon. Mais c'est seulement en 1953, après un voyage à Stalingrad organisé par l'Union des écrivains, qu'il réussit à trouver une forme adéquate avec Die Fahrt nach Stalingrad. Ce texte fut pour lui une "délivrance ", lui permettant de «se libérer de quelque chose qu'il avait sur le cœur » et qui s'exprimait avec " exubérance ${ }^{9}$ ». Une dizaine d'années après la fin de la guerre, émerge une nécessité individuelle de se confronter au passé par l'écriture, nécessité dont témoigne le nombre croissant des textes publiés depuis 1955 et la création de collections propres dans certaines maisons d'édition (par exemple « tangenten » au Mitteldeutscher Verlag Halle) ${ }^{10}$. Ce faisant, ces dernières tablaient également sur la valeur marchande de ces textes censés rencontrer un large accueil dans la population ${ }^{11}$.

En 1956, le IV congrès de l'Union des écrivains se penche enfin sur cette question. Anna Seghers déplore que la Seconde Guerre mondiale ne soit pas abordée dans la littérature est-allemande. Celle-ci s'était trop concentrée sur la représentation du présent, alors que « chaque morceau de la vie qui n'est pas encore compris jusque dans les moindres détails demande à être façonné, afin que l'on comprenne entièrement le 
présent $^{12} »$. Seghers souligne qu'il ne suffit pas de mettre en scène des héros antifascistes, mais qu'il faut aussi des romans qui disent « ce que vivent des jeunes gens qui, séduits par le fascisme, sont partis à la guerre, leurs doutes et leur désespoir, le changement provoqué par leurs lourdes expériences ${ }^{13}$ ». Ludwig Renn, de son côté, s'étonne que «l'expérience collective dominante de toute une génération » ne soit pas vraiment présente dans la littérature. Il réclame « la représentation de l'expérience de la guerre du point de vue de ceux qui l'ont conduite militairement, du point de vue de ceux notamment qui l'ont faite du mauvais côté et pour qui elle a provoqué un examen intérieur, une prise de conscience et une conversion ${ }^{14} »$. Comme Seghers, Renn estime que la littérature ne peut se limiter à décrire ceux qui étaient du "bon côté", il souligne la nécessité de thématiser l'attirance qu'a exercée le national-socialisme sur les jeunes, d'expliquer les causes de la guerre et de montrer la « conversion » positive des individus.

Ces prises de position en faveur d'une littérature sur la Seconde Guerre mondiale sont à double tranchant. D'un côté, Seghers et Renn semblent reconnaître les erreurs d'une politique culturelle qui orientait la littérature trop exclusivement sur des sujets contemporains, ils sont conscients que l'on ne peut pas construire une société en excluant du discours public l'expérience collective dominante qu'était la guerre et ils reconnaissent que le point de vue strictement antifasciste n'est pas en mesure de satisfaire les attentes des lecteurs. D'un autre côté, ils donnent une orientation aux représentations futures de la guerre en se conformant au discours dominant : la part de responsabilité notamment de la jeune génération de soldats est minimisée (c'est une génération séduite); de plus il s'agit de montrer comment s'est opérée la transformation intérieure des anciens soldats, facilitant leur récupération morale et leur intégration au sein de la société.

11 La teneur hautement politique de ces prises de position s'exprime par ailleurs dans une remarque de Ludwig Renn évoquant «une littérature à caractère pacifiste naïve et éloignée des faits $»^{15}$ : il utilise ici un mot-clé de l'idéologie des années cinquante. En effet, dès la fin des années quarante, sous l'influence des autorités soviétiques, le discours introduit la distinction entre guerre "juste " et "injuste», distinction permettant de justifier une guerre de défense du monde socialiste. Dans ce contexte, la représentation des souffrances et des cruautés de la guerre qui, ne pouvant être porteuses de sens, provoquaient une condamnation nette de la guerre, fut considérée avec suspicion. La " perspective des tranchées » fut régulièrement la cible de la critique littéraire ${ }^{16}$. Cette position contre le pacifisme était d'autant plus nécessaire qu'en 1956 fut créée l'armée est-allemande (NVA) et qu'on demanda à des écrivains tout juste passés « du soldat hitlérien au combattant pour la paix », d'aller « enthousiasmer [la] jeunesse pour [...] l'art de la guerre $»^{17}$.

12 La littérature sur la Seconde Guerre mondiale qui a émergé à la suite du IV congrès des écrivains offre un panorama varié de publications. Si les auteurs, comme le remarque Ursula Heukenkamp, ne s'appliquent pas à donner, comme on l'exigeait d'eux, une image héroïque de la guerre et s'ils continuent à représenter «la guerre comme un enfer ", ils introduisent en même temps le leitmotiv de la " conversion » pour signifier qu'une issue idéologique devient possible pour eux ${ }^{18}$. D'autres auteurs en revanche, et notamment les plus jeunes, se retrouvent vite attaqués par une critique littéraire qui leur reproche une "écriture dure» (harte Schreibweise), influencée par les modèles américains (Ernest Hemingway, Norman Mailer). Cette notion vague d'« écriture 
dure» permettait de rejeter aussi bien le naturalisme documentaire dans les descriptions des atrocités de la guerre qu'une perspective psychologisante ou que le choix d'une narration à partir d'un personnage "négatif ». On condamnait également le manque de positionnement moral des auteurs ou l'absence du motif de la « conversion $»^{19}$.

La discussion autour de la littérature de guerre en RDA dans les années cinquante montre bien les failles qui existaient dans les directives de la politique culturelle: encouragée par les autorités à entamer une confrontation avec le passé et de récupérer le sujet pour le présent, l'écriture des expériences de guerre rencontra un écho important auprès des écrivains concernés. Mais, comme le montrera l'exemple de Fühmann, la réalité que ces derniers choisissent de représenter, les moyens d'expression et l'esthétique ne correspondent pas à la vision officielle. Trop focalisée sur le passé et ne se souciant pas d'établir des liens avec le présent socialiste, cette littérature finit par saper et remettre en question le consensus antifasciste qui avait pour objet de déculpabiliser la population est-allemande. Du point de vue officiel, il était alors dans la logique des choses qu'en 1959 la conférence de Bitterfeld réaffirmât la nécessité pour les écrivains de se consacrer à des sujets d'actualité et d'œuvrer pour un lien plus étroit entre ouvriers et artistes. Suite à cette conférence, une rencontre du bureau de l'Union des écrivains avec des jeunes auteurs sur le "style dur » s'est vite transformée en «tribunal » destiné à empêcher l'écriture d'autres textes de ce type ${ }^{20}$.

\section{La trilogie sur la guerre de Franz Fühmann}

1955, Franz Fühmann publie son premier texte en prose aux éditions Aufbau. Kameraden $^{21}$ est le fruit d'un travail intense entamé deux ans auparavant et ayant bénéficié des conseils de Max Schroeder, lecteur chez Aufbau. Rétrospectivement, Fühmann présente la nouvelle comme sa première œuvre, alors qu'il avait déjà publié de la poésie ${ }^{22}$. En 1957 suivent deux nouvelles, Das Gottesgericht et Kapitulation ${ }^{23}$ qui, par leur proximité thématique et narrative, peuvent former une trilogie ${ }^{24}$.

Fühmann a choisi la forme de la nouvelle, apparemment de façon intuitive, dans le souci d'écrire quelque chose qui «devait être lu» et qui «se devait donc d'être captivant ${ }^{25} »$. La proximité de la nouvelle et du drame permet effectivement de capter l'attention du lecteur. L'effet cathartique du drame se traduit dans la nouvelle par une tendance à la "moralité ", ce qui peut expliquer le recours à ce genre littéraire dans des périodes de changement et de transformation où l'on " tire les leçons » du passé. Tel était bien le souci de Fühmann à cette époque : «Je n'écrivais pas pour faire une expérience, mais pour exprimer mes expériences et mon savoir; chaque récit devait avoir un fabula docet, et chaque poème était une ancilla ideologiae ${ }^{26}$. "

16 Contrairement à ce que ces propos de Fühmann pourraient laisser craindre, contrairement à certains poèmes antérieurs et contrairement aussi à d'autres textes qui vont suivre, ce didactisme librement choisi ne s'exprime pas de façon pesante dans les trois nouvelles. Les nouvelles, écrites du point de vue exclusif de soldats allemands impliqués dans différents crimes de guerre et se terminant par la mort des personnages principaux, n'ont aucun caractère de conciliation. Elles ne suggèrent aucune perspective d'avenir dans le sens d'une possible «conversion» et se limitent à démontrer les conséquences de l'endoctrinement par l'idéologie nationale-socialiste. 

des personnages. Ainsi se trouve-t-on respectivement en juin 1941 dans une compagnie stationnée dans le territoire de Memel, à l'extrême pointe de la Prusse orientale (Kameraden), en mai 1943, en Grèce, auprès d'une compagnie de transmission d'informations (Das Gottesgericht) et le 9 mai 1945, "sept heures après la capitulation sans conditions du haut commandement de la Wehrmacht ${ }^{27}$ » (75), dans la forêt de Bohème (Kapitulation). Tous les protagonistes sont des soldats de la Wehrmacht, de rangs divers, qui vont être impliqués dans des crimes commis en dehors des combats, dans des situations quelque peu atypiques qui constituent l'élément central de la nouvelle en tant que genre. Fühmann ne dépeint pas la réalité de la guerre sous l'aspect de sa cruauté matérielle, comme le font certains des romanciers critiqués pour leur « style dur ", il met l'accent sur la dimension psychologique de ses personnages, sur les conflits intérieurs qui les animent, une approche qui ne fut pas moins condamnée par la critique littéraire de la RDA.

Les conflits naissent dans des situations inhabituelles. Dans Kameraden, trois soldats tuent accidentellement la fille du commandant de leur compagnie; la décision de dissimuler ce meurtre met à l'épreuve leur «esprit de camaraderie » dont Fühmann déconstruit le mythe volontairement entretenu dans la Wehrmacht et en train de ressurgir dans le discours public d'après-guerre ${ }^{28}$. Das Gottesgericht met en scène quatre soldats qui, envoyés en reconnaissance sur une plage à la recherche de partisans, tombent sur le cuisinier grec (Agamemnon) de la compagnie qui se trouve là bien que ce soit interdit. La focalisation passe alors par le point de vue des différents personnages (la victime potentielle et ses bourreaux). Les réflexions contradictoires des uns et des autres ainsi que le ralentissement du rythme du récit qui s'ensuit augmentent le suspense. Dans Kapitulation, un sous-lieutenant de la SS et deux soldatspoliciers sont sur le point de pendre un jeune déserteur, un jour après la capitulation, mais l'approche de soldats de l'Armée rouge les fait fuir. La nouvelle décrit la tentative de survivre du jeune soldat, tiraillé entre la volonté de se rendre à l'Armée rouge et celle de rejoindre ses « camarades » et de continuer à combattre.

On a reproché aux nouvelles de Fühmann de suivre un schéma simpliste entre le bien et le mal où chaque personnage était soit positif soit négatif. On a voulu voir dans cette confrontation entre le bien et le mal l'expression du «typique », catégorie du réalisme socialiste en vogue dans les années cinquante ${ }^{29}$. Ce jugement est presque devenu un topos dans certaines études sur Fühmann; il est repris, voire amplifié, sans être interrogé. Mais il n'en est rien, la conception des personnages est bien plus complexe (ce qui fait toute la force des récits), leur rapport à la doctrine du réalisme socialiste aussi $^{30}$.

Dans Kameraden, Fühmann dessine trois caractères très différents. Le caporal-chef Karl W. est un ancien membre du Freikorps, il a l'habitude de tuer et n'est pas spécialement affecté par la mort de la fille du commandant. Josef L., l'intellectuel, est un fervent national-socialiste, fils d'un haut dignitaire nazi, pour qui la vie d'un homme ne vaut pas beaucoup. Le personnage de Thomas P., âgé de dix-huit ans comme Josef, reste relativement flou. Seul à avoir des remords, il se trouve aux prises avec un conflit de conscience entre la dénonciation du crime et la solidarité avec ses camarades qui lui a été inculquée dans un camp d'entraînement des jeunesses hitlériennes. Si Thomas est le seul personnage qui a manifesté un certain sens moral, son endoctrinement idéologique ne fait toutefois pas de doute. Lorsque Karl parle de ses combats contre les 
communistes à Berlin, Thomas n'y voit pas d'inconvénient : «Et la Commune, au fond, ce ne sont pas des êtres humains. Je veux dire - comme aujourd'hui... » (24). Lorsque, avec l'aide du père de Josef, le meurtre de la fille est imputé aux Russes, ce qui permet en même temps d'attiser la haine des soldats quelques heures avant l'invasion de l'Union soviétique, Thomas l'approuve. Ce n'est que plus tard, lorsque la compagnie entre en Lituanie et que le commandant décide de pendre deux jeunes filles dans un petit village en représailles de la mort de sa fille que la conscience de Thomas s'éveille. Il se sent coupable de la mort des deux filles et veut prendre à son compte le meurtre. La nouvelle se termine sur une tentative de fuite de Thomas qui sera abattu par ses camarades : «Il entendit les balles siffler, et ensuite un feu a traversé ses poumons. Il s'évanouit et resta par terre, dans une mare de sang. Des paysans lituaniens le trouvèrent" (48). Thomas est un personnage tiraillé par des sentiments contradictoires, on ne peut pas voir en lui le «bon » personnage montré en exemple contre les «méchants » fascistes ${ }^{31}$. Dans la nouvelle, sa désertion témoigne d'un réveil de conscience et d'une action en conséquence, mais aucune issue positive n'est proposée. À l'instar des deux récits qui suivront, le personnage mourra de toute évidence de ses blessures ${ }^{32}$.

Dans Das Gottesgericht, les personnages sont également présentés de façon ambiguë. Désignés selon leur hiérarchie par D. (adjudant), C. (sergent), B. (caporal-chef) et A. (opérateur radio), ils se retrouvent dans un face à face de quelques minutes avec le cuisinier grec Agamemnon. Le retournement progressif des valeurs associées aux personnages donne toute sa tension au récit. Au lieu de tuer immédiatement le cuisinier, D., professeur d'histoire au lycée et spécialiste du Moyen Âge, propose d'appliquer une coutume moyenâgeuse, le jugement de Dieu, qui combinerait «le devoir, le droit et la générosité » (55). Si le lecteur croit pouvoir y déceler un certain sens de la justice et un reste d'humanité, il apprend plus loin que D. est un fervent adepte du national-socialisme qui voudrait prendre le Moyen Âge comme modèle pour une Allemagne nouvelle. Le sergent $C$., le seul personnage d'emblée négatif, est contre cette expérience, craignant de passer à côté d'une « aventure bienvenue » dans sa base militaire où, depuis trois ans, rien ne se passe. Il réclame une compensation pour sa mission et veut voir «un peu de sang coul[er]» (55). B. propose d'abord de tuer Agamemnon, il sera le premier à tirer sans y parvenir pour autant. Il prend subitement conscience de la gravité de son action et il voit surgir des images de son enfance qui lui rappellent les combats dans un quartier ouvrier, les morts et son père emporté dans un camion. Pourtant la nouvelle ne bascule pas, comme on pourrait l'attendre d'un récit résolument "socialiste », vers la prise de conscience d'un fils d'ouvrier qui s'est engagé du mauvais côté. Si B. est gagné par un sentiment de honte à cause d'un combat qui n'était pas « honnête ", il espère toutefois avoir gagné l'estime du sergent. L'opérateurradio A. apparaît au début comme celui qui prendra la défense du cuisinier. L'idée de B. de le tuer l'effraye. Mais étant au plus bas de la hiérarchie, A. ne parle pas, tout se passe dans sa tête. Et peu à peu ce personnage aux pensées apparemment humaines est gagné par un sentiment de supériorité : «[...] et d'un coup il pensa qu'il n'avait qu'à bouger une phalange, et cet homme serait couché là, anéanti, devant lui. 'Mais je n'ai qu'à le vouloir !', pensa-t-il, 'il suffit d'un éclair de ma volonté et sa vie est fichue !' » (62). Il se révèle imprégné comme les autres par l'idéologie national-socialiste, se voyant, grâce au Führer, en «maître sur la vie et la mort » et en « Dieu » (63). C'est A. qui finalement tue Agamemnon. 
22 Dans la dernière nouvelle, Kapitulation, les angoisses et tourments du personnage principal, un jeune déserteur sur le point d'être pendu, constituent le centre du récit. Fühmann s'est inspiré ici d'une nouvelle de l'Américain Ambrose Bierce, "An Occurrence at Owl Creek Bridge » (1890) qui relate les derniers moments d'un pendu qui s'imagine que la corde se rompt et qu'il s'en sort ${ }^{33}$. Les pensées du jeune soldat, ses visions et son imagination, caractérisant son point de vue narratif, auxquelles s'ajoute la présence concrète de la corde, s'avèrent être des éléments intertextuels empruntés à Bierce dont les textes décrivent souvent des situations limites.

Le soldat est d'abord présenté comme une victime de ses persécuteurs. Lorsque ceux-ci s'enfuient devant les soldats de l'Armée rouge, il se retrouve en liberté, désorienté et désemparé. L'action se déroule dans un espace très limité (une clairière au-dessus d'un ravin, symbole de l'abîme psychologique du personnage). Lorsque le déserteur aperçoit ses persécuteurs en bas de la pente, il voudrait voir «le visage de ses meurtriers", " pour graver dans sa mémoire leurs traits maudits ", afin de pouvoir les peindre : « Les trois visages du Cerbère aux trois têtes, du chien de garde dans l'enfer de la guerre, c'est ainsi qu'il voulait les peindre, et dans son imagination, le tableau était déjà prêt, excepté les trois taches vides : les visages inconnus de ses meurtriers. » (81) Mais cette dimension victimaire du personnage est relativisée. On comprend que la désertion n'a lieu qu'à la toute fin de la guerre, qu'il ne s'agit pas d'une décision consciente, mais d'un geste de panique. Par ailleurs, le comportement du soldat face à la présence de soldats russes témoigne de l'endoctrinement idéologique qu'il avait subi très jeune - il se demande si ce sont bien des hommes. Attiré un instant par l'idée de se rendre - un possible point de retournement de la nouvelle - il y renonce, conscient des crimes commis par la Wehrmacht et craignant la vengeance de l'Armée rouge (la description d'une Armée rouge vindicative ne correspondait guère à la vision officielle). Le soldat s'accroche alors subitement à l'idée de camaraderie, de nécessaire cohésion des Allemands, voire de poursuite du combat. Il va jusqu'à admettre le bien-fondé de sa pendaison pour avoir trahi ses camarades et jeté son arme. Comme dans les autres nouvelles, le personnage est ambigu. Il est psychiquement perturbé et représente tout sauf un modèle d'identification. Et la nouvelle se termine de fait par sa mort.

La critique littéraire de RDA déplore, dans les nouvelles de Fühmann, le manque d'ancrage dans le présent de la société socialiste, ce qu'elle formulait comme un défaut d'« orientation dans l'ici et le maintenant », elle soulignait l'absence "d'instructions pour agir » permettant au lecteur de « dépasser véritablement le passé fasciste ${ }^{34}$ ». Un narrateur qui n'apporte aucun commentaire ni ne prend position sur les actions des personnages mais qui s'attache essentiellement à leurs pensées et à leurs sentiments est en décalage avec le système de représentation littéraire du réalisme socialiste promu par la doctrine officielle.

Fühmann refuse ostensiblement d'« éduquer » le lecteur. L'auteur n'établit pas de liens avec le présent, avec la nouvelle société. Il limite volontairement la perspective de ses nouvelles qui se terminent par la mort des principaux personnages. En centrant la thématique de ses écrits sur leurs réflexions, leurs déchirements intérieurs, leurs émotions et tourments psychiques, il met en avant, avant tout autre chose, les conséquences de l'endoctrinement idéologique et l'incapacité des personnages à s'en libérer. Les sursauts d'humanité ne renvoient pas à un possible changement. Contrairement à leur auteur qui affirme avoir vécu une « conversion » dans le camp de rééducation soviétique et qui essaye d'en témoigner dans des textes autobiographiques, 
les nouvelles ne reprennent pas ce topos ${ }^{35}$ dont l'auteur dira encore vingt ans plus tard, dans son journal de Hongrie, qu'il n'a pas encore réussi à vraiment la décrire.

En revanche, comme l'affirme Stephan Hermlin, Fühmann a réussi à « faire apparaître les grandes questions de l'époque derrière les péripéties d'un cas individuel », son activité littéraire est décrite comme une "autocritique nationale ${ }^{36}$ ». Certains ont souligné la proximité des personnages avec leur auteur, ils seraient « un médium de l'auto-accusation et de l'autopunition ${ }^{37} »$. Mais le fait d'avoir choisi un genre fictionnel et des personnages souvent anonymes signifie que c'est à toute une génération qu'il s'adresse. On passe d'un aveu de culpabilité personnelle exprimé dans Die Fahrt nach Stalingrad à une accusation plus large des soldats de la Wehrmacht.

Car les trois nouvelles mettent en évidence des crimes perpétrés par des soldats et posent la question de la culpabilité individuelle à un niveau plus large que celle du seul auteur. Ainsi, Kameraden évoque clairement la guerre d'invasion meurtrière en Union soviétique, les victimes civiles et les exactions commises sur le front de l'Est. Das Gottesgericht met en scène l'assassinat, sans raison, d'un civil grec et, par là, la disposition de simples soldats à tuer, voire leur envie de tuer pour s'offrir une distraction. Dans Kapitulation, le soldat déserteur a, à deux reprises, une vision, celle d'un vieillard à la barbe blanche et au visage rouge et bleu, pendu dans un village d'Ukraine lors de la retraite allemande et victime de la politique de la terre brûlée. Le jeune soldat clame son innocence personnelle, il dit qu'il n'est pas coupable, mais « il ne pouvait plus croire son cœur.» (91) Le lecteur non plus. Une autre vision («allées sur allées, les arbres innocents avec le poids des pendus ») témoigne de l'ampleur des crimes commis sur le front de l'Est.

Avec cette évocation des différents crimes de la Wehrmacht, Fühmann va à l'encontre non seulement du discours antifasciste prêt à "racheter » une population qui s'est compromise, mais il se positionne aussi à contre-courant d'un discours social qui, dans les années cinquante, minimise les crimes de la Wehrmacht et présente les soldats comme des victimes. Contrairement à l'immédiat après-guerre où les procès de Nuremberg, suivis par l'opinion publique, mettaient au grand jour les crimes de guerre et les crimes contre l'humanité commis par la Wehrmacht, l'image change peu à peu au cours des années cinquante où naît le mythe d'une "armée propre ${ }^{38}$ ». L'émergence d'une culture des vétérans, le rejet de la «justice des vainqueurs» et le fait que les Alliés privilégiaient la condamnation des grands criminels de guerre, laissant ainsi penser que les simples soldats étaient des "objets désarmés ${ }^{39}$ ", voire des victimes, allaient dans ce sens, notamment en RFA. De plus, dans les deux États allemands, la politique de remilitarisation joua un rôle important. Si le taux d'enrôlement effectif d'anciens officiers de la Wehrmacht dans la nouvelle armée est-allemande fut moins important qu'en RFA, le discours officiel mettait avec insistance sur le compte du "régime fasciste» les exactions de l'armée et tendait ainsi à déculpabiliser individuellement les soldats ${ }^{40}$. Par ailleurs, la politique d'intégration menée par le SED, consistant à accepter dans ses propres rangs d'anciens membres du NSDAP, s'est accompagnée de leur rapide réhabilitation, en dépit des protestations venant aussi bien du côté des victimes du national-socialisme (l'Association des persécutés du régime nazi, VVN) que d'autres partis comme le CDU et le LDPD ${ }^{41}$.

29 Il faut donc également lire les nouvelles de Franz Fühmann dans ce contexte de réhabilitation des anciens soldats et des membres du NSDAP, un contexte qui favorise la déculpabilisation individuelle et l'oubli afin d'intégrer de larges parties de la 
population dans la nouvelle société. C'est une fois de plus la littérature qui prend en charge la mémoire collective pour rappeler, dans le cas de Fühmann, les actions criminelles de la Wehrmacht et la responsabilité individuelle de chaque soldat.

\section{Le passage de l'expérience vécue à la mémoire}

Le recueil Stürzende Schatten (1959) contient, en dehors des nouvelles Das Gottesgericht et Kapitulation, un cycle de trois récits très courts intitulé Das Erinnern. Si les nouvelles sur la guerre sont écrites dans la perspective de l'expérience immédiate et ne dépassent pas le cadre temporel de la guerre, les trois récits regroupés dans Das Erinnern sont conçus dans la perspective du souvenir.

Traum 1958 est inspiré par l'actualité politique, la résolution adoptée par le Bundestag fin mars 1958 d'équiper la Bundeswehr d'armes nucléaires. À partir de cette décision largement contestée dans l'opinion publique, Fühmann esquisse un scénario de l'horreur en ravivant la mémoire de la Seconde Guerre mondiale. La trame du rêve, avec ses abondantes associations et images, lui permet de mêler les visions d'une guerre terminée avec celle d'une guerre à venir. Le personnage principal, le boulanger Hans K., fait un rêve dans son rêve. Il fait souvent des cauchemars sur la fin de la guerre. Membre du Volkssturm à l'âge de quinze ans, il est décoré par Hitler dans son bunker - cette image de Hitler décorant de très jeunes garçons est entre-temps devenue un cliché utilisé encore récemment dans le film Der Untergang. Fühmann décrit un spectacle grotesque de vieillards lourdement décorés et d'un Führer qui n'a plus de visage et qui les assaille de sons qui ressemblent au bruit d'une mitrailleuse digne d'Ernst Jandl ${ }^{42}$. À ce rêve se superpose un deuxième rêve où le Führer et les généraux réapparaissent, "vieillis d'une douzaine d'années» (107). Dans le personnage du " chancelier » et du "ministre de la défense ", le lecteur reconnaît facilement Konrad Adenauer et Franz-Joseph Strauß en train de vanter l'arme atomique. Le rêve de Hans K. a mêlé ses souvenirs de guerre et ses peurs d'une guerre atomique. Il revient au narrateur de suggérer que c'est une conversation avec un client qui est à l'origine de ce rêve particulièrement chargé, elle-même "provoquée par la nouvelle sur l'armement atomique de la Bundeswehr qui fait dire à Hans K. qu'un boulanger doit s'occuper de faire du pain, et non de politique [...]» (112).

La dimension pédagogique de ce récit et l'intention de dénoncer la politique d'armement ouest-allemande manquent de nuance. La résurgence du Führer en chancelier de l'Allemagne fédérale participe d'un engagement politique qui nuit à la crédibilité littéraire. En plus de ce dérapage dans la lecture de l'histoire, Fühmann avait reconnu lui-même avoir raté la forme-même de ce récit, en se laissant trop guider par des circonstances extérieures. Ce qu'il faut toutefois souligner, c'est le recours par Fühmann au récit d'un rêve, un type de récit inspiré des avant-gardes et du "formalisme ", alors que l'on est encore en pleine période de promotion du réalisme socialiste.

Le deuxième récit, Grenzstation, rend compte des craintes et des peurs chez les peuples voisins de l'Allemagne marqués par la période nationale-socialiste. Le personnage principal, le tchèque Dr. L., voyage en train en direction de l'Allemagne. À l'approche de la gare frontalière, il se souvient de son dernier voyage en Allemagne, douze ans plus tôt, où il fut conduit dans un wagon à bestiaux vers une usine de Berlin, comme «travailleur forcé ». Il revoit en pensée l'usine et les baraquements, les colonnes de 
soldats hurlant des chants nazis, le surveillant à la moustache hitlérienne qui l'insulte et le menace à l'approche du capitaine. Ce capitaine entre brutalement dans le compartiment où est assis le Dr. L. qui est pris d'un malaise en voyant «cet uniforme [qui] se tenait là imposant et gris et immobile» (114), avant de comprendre que l'uniforme est celui d'un agent des douanes de la RDA.

Fühmann recourt ici à une expérience rapportée par son collègue et ami, l'intellectuel tchèque Ludvík Kundera. Dans une lettre à Kundera, Fühmann lui parle de ce récit dont il est le personnage principal: "Le thème était constitué par votre récit sur les sentiments et les pensées qui se sont emparés de vous lorsque vous avez vu pour la première fois les uniformes de notre armée. ${ }^{43}$ " En effet, lors de la création de son armée en 1956, la RDA dut choisir des uniformes qui se distinguaient aussi bien de ceux de la Bundeswehr (suivant la mode américaine) que de ceux de l'Armée soviétique (par souci de donner une image d'indépendance). On opta alors pour la continuation de «l'héritage national » de la tradition militaire allemande, en reprenant le modèle des uniformes de la Reichswehr qui avaient également servi à la Wehrmacht ${ }^{44}$. Ce deuxième récit du cycle Das Erinnern est donc également le fruit d'une réflexion sur le présent immédiat. Fühmann évoque, avec l'expérience traumatisante du travail forcé, l'un des crimes du régime national-socialiste. Il se livre également à une critique implicite de la réutilisation des symboles militaires du nazisme par la RDA.

Le cycle se termine par Über den Waldsee, un récit qui culmine dans un dialogue sur la guerre entre un père et sa jeune fille. Ceux-ci se trouvent en panne d'essence lors d'un tour en bateau à moteur, et ils se font aider par une famille russe. L'enfant demande ce que font les Russes en Allemagne ; le père parle «des hommes méchants qui ont fait une guerre contre des hommes bons" (117) et des Russes qui ont aidé les bons. À la question de savoir s'il avait également été soldat, s'il avait été un mauvais ou un bon, le père répond d'abord par un silence, puis par un mensonge : “'Tu as été un bon soldat, papa?' demanda l'enfant. 'Oui', dit l'homme à la voix éteinte. [...] 'Fallait-il que je mente?' pensa l'homme plein d'amertume " (118). L'homme se demande "pendant combien de temps ces années maudites hanteront encore notre vie " et le récit se conclut sur cette réflexion : «Que le mensonge meure avec notre génération ! (118).

Ce texte très court est significatif à plusieurs niveaux. Le mensonge du père renvoie à un sentiment de culpabilité non avoué qui, étendu à tous ceux qui en font l'expérience, confronte le lecteur à sa propre implication dans les crimes - on retrouve l'un des thèmes des nouvelles sur la guerre. Par ailleurs, le texte pose la question de l'impossibilité de transmettre d'une génération à l'autre l'expérience de la guerre en raison du sentiment de honte lié à celle-ci. Fühmann stigmatise le silence de toute une génération ainsi que le refoulement de la culpabilité, et il souligne l'enfermement fatal dans le mensonge: si seulement la mort naturelle de toute une génération peut $\mathrm{y}$ mettre fin, cela sous-entend que toute une génération renonce à un travail de confrontation avec le passé. Le récit condamne le refoulement du passé dans les deux États allemands, sa dimension critique a néanmoins un impact particulier dans le contexte de la RDA. Car dans la perspective mémorielle du cycle Das Erinnern, affirmer que la transmission est un mensonge revient à critiquer la réintégration des anciens partisans du national-socialisme par le nouveau régime, à travers l'adhésion à l'antifascisme. l'immédiateté de la guerre, témoignent de la difficulté à effectuer le passage du passé 
au présent, présent que l'auteur pense maintenant en termes de transmission: transmission de l'expérience des horreurs de la guerre afin d'empêcher une nouvelle guerre (à cet égard, le récit Traum 1958 devrait être taxé de pacifisme) ; transmission des expériences des victimes du national-socialisme dans Grenzstation; impossible transmission de génération à génération dans Über den Waldsee. Or, on constate que cette perspective de la mémoire est étroitement liée au présent dans lequel elle s'inscrit. La mémoire étant perméable aux exigences du présent, le discours littéraire qui la véhicule est aussi sensible à ces exigences. Ainsi, les trois récits de Fühmann sont-ils ponctués d'emprunts au discours officiel, que ce soit sur la RFA comme unique héritière du Troisième Reich et le discours de la guerre froide dans le premier récit, le discours élogieux sur les réalisations récentes de «l'autre Allemagne » dans le deuxième, ou encore le discours sur l'amitié germano-soviétique dans le troisième. Toutefois, cela n'empêche pas qu'il y ait parallèlement un discours critique sur l'armée est-allemande ou, plus significatif, sur l'antifascisme. provoqué un tournant dans sa vie, il mentionne « la vérité sur Auschwitz » en donnant quelques précisions. Selon lui, Auschwitz est à comprendre comme un «terme générique » rassemblant " la totalité de la théorie et de la pratique des crimes contre l'humanité du national-socialisme qui, précisément en tant que totalité, n'était pas connue par la majeure partie de la nation avant $1945 »^{46}$. «Auschwitz » n'est donc pas à comprendre ici seulement comme synonyme de l'extermination des Juifs, mais de tous les crimes du régime national-socialiste. Dans ses nouvelles sur la guerre, la thématique du génocide n'apparaît pas, alors qu'il évoque entre autres les crimes sur le front de l'Est. Ceci peut s'expliquer par la perspective choisie qui est celle des soldats (avec une tendance à la généralisation), permettant de décrire des expériences faites par une majorité et excluant par là-même la question des camps d'extermination. Par ailleurs, Fühmann reste fidèle à la reconstitution de l'univers mental d'un soldat pendant la guerre qui, sous l'influence de l'endoctrinement idéologique, n'a guère commencé à se confronter au génocide. En revanche, le sujet aurait pu être traité dans les récits « mémoriels » du cycle Das Erinnern, ce qui n'est pas le cas.

Fühmann évoque le génocide ailleurs, dans ses écrits à caractère autobiographique. Dans Die Fahrt nach Stalingrad (1953), il décrit un SS que «le Führer en personne a nommé commandant du camp d'extermination » et qui lâche une meute de chiens sur une «foule de gens qui est en route pour la mort dans le crématoire ». Il évoque « les piliers d'où s'échappe le gaz [qui] sont joliment déguisés en colonnes attiques ${ }^{47}$ ". Cette première évocation des chambres à gaz est immédiatement suivie d'une remise en question personnelle, à travers le «nous » générationnel de ce texte : «Ah, nous ! Nous avons / des mains si pures, des mains si innocentes, / nous n'avons jamais été 
commandant de camp. [...]», et un peu plus loin, « Nous n'avons pas / nous-mêmes donné de coups aux corps, non, nous n'avons fait que regarder / nous avons juste laissé faire. Nous montions uniquement / la garde devant ces tueurs. [...]». Ce passage indique dès 1953 une prise de conscience de la culpabilité dans le génocide. Exprimée à cette époque encore à la première personne du pluriel, elle sera entièrement assumée par le « je » de l'auteur dans le Journal Vingt-deux jours ou La moitié de la vie vingt ans plus tard. Dans l'intermédiaire, Fühmann écrira au début des années soixante les récits autobiographiques de Das Judenauto. Ce récit qui a donné le titre au recueil est une confrontation de l'auteur avec son propre antisémitisme, une entreprise difficile à cause de la "résistance de la mémoire " perceptible dans cette tentative « d'édifier le monument de sa honte ${ }^{48} »$. Le point de vue autobiographique lui est donc à présent nécessaire pour revisiter sa conscience et ses préjugés, pour remonter aux origines d'un antisémitisme aux conséquences exterminatrices à l'intérieur de lui-même, ce qui correspond à un nouveau degré de la conscience d'avoir été coupable pour avoir laissé faire.

Les récits des années cinquante sont une première étape dans ce sens. Leur intérêt, dans le contexte de l'époque, est d'avoir attiré l'attention sur la culpabilité personnelle de chacun et d'avoir rappelé les crimes commis par les soldats de la Wehrmacht, alors qu'il régnait au sein de la société un consensus sur un oubli volontaire permettant l'intégration des soldats. Si les nouvelles et récits de Franz Fühmann sont instructifs sur la manière dont la littérature de RDA se confronte au passé national-socialiste dans les années cinquante, leur réexamen dans le contexte actuel des interrogations sur la mémoire du national-socialisme n'est pas moins éclairante. Ainsi, face aux débats sur les crimes de la Wehrmacht qui ont ressurgi dans le sillage de l'exposition organisée par le Hamburger Institut für Sozialforschung, il est important de voir que, dès les années cinquante, un auteur comme Fühmann a déconstruit le mythe d'une Wehrmacht "propre ». De même, face à la critique et la remise en question de l'antifascisme après 1989, il est intéressant de voir qu'on pouvait questionner ce discours, même à une époque sensible comme les années cinquante. L'exemple des récits de Fühmann montre que la littérature réussissait à se situer à contre-courant du discours officiel, voire à le remettre sérieusement en question et à maintenir présente la mémoire du passé dans un contexte d'oubli volontaire.

\section{NOTES}

1. Franz Fühmann: Vingt-deux jours ou La moitié de la vie, Traduit de l'allemand par Philippe Préaux, Paris, Flammarion, 1988, p. 169. Traduction légèrement modifiée. Par la suite sigle Vdj suivi des numéros de page.

2. Franz Fühmann : «Antwort auf eine Umfrage », in Id. : Wandlung, Wahrheit, Würde. Aufsätze und Gespräche 1964-1981, Darmstadt, Luchterhand, 1985, p. 17-21, ici p. 20.

3. Ibid.

4. Cf. Wolfgang Emmerich: «Die Risiken des Dafürseins. Optionen und Illusionen der ostdeutschen literarischen Intelligenz 1945-1990 ", in Sven Hanuschek/Therese Hörnigk/ 
Christine Malende(dir.): Schriftsteller als Intellektuelle. Politik und Literatur im Kalten Krieg, Niemeyer, Tübingen, 2000, p. 275.

5. Cité dans Leonore Krenzlin : «Theoretische Diskussionen und praktisches Bemühen um die Neubestimmung der Funktion der Literatur an der Wende der fünfziger Jahre », in Ingeborg Münz-Koenen (dir.) : Literarisches Leben in der DDR 1945-1960. Literaturkonzepte und Leseprogramm, Akademie-Verlag, Berlin, 1979, p. 152-195, ici p. 161.

6. Ursula Heukenkamp : «Helden, die einer besseren Sache wert gewesen wären... Kriegsprosa in der DDR der fünfziger Jahre », in Hans Wagener (dir.) : Von Böll bis Buchheim : Deutsche Kriegsprosa nach 1945, Amsterdam, Atlanta, Rodopi, 1997, p. 365-387, ici p. 368.

7. Ibid., p. 372.

8. Ludwig Renn : «Weshalb keine Literatur über den Krieg? », in Neue Deutsche Literatur, 1/1956, p. 126-128, ici p. 127.

9. Josef-Hermann Sauter : «Interview mit Franz Fühmann », in Weimarer Beiträge 1/1971, p. 33-53, ici p. 39sq.

10. Cf. Martina Langermann: "Diskussionen um die "harte Schreibweise“. Eine Reaktion auf literarische Entdeckungen junger Autoren?», in Weimarer Beiträge, 9/1990, p.1419-1429, ici p. 1423. Pour n'en donner que quelques chiffres : sur les 97 textes sur la guerre (romans et récits) publiés entre 1949 et 1960, une vingtaine seulement date d'avant 1955. Cf. Olaf Lange: "Wandlung oder Tod. Der zweite Weltkrieg in der Prosa der DDR (1949-1960)", in Ursula Heukenkamp (dir.) : Unerwünschte Erfahrung. Kriegsliteratur und Zensur in der DDR, Berlin, Weimar, Aufbau-Verlag, 1990, p. 100-134.

11. Cf. Olaf Lange : «Wandlung oder Tod... », op. cit., p. 128 et 130.

12. Anna Seghers: "Die große Veränderung und unsere Literatur. Rede auf dem IV. Schriftstellerkongress ", in Id. : Aufsätze, Ansprachen, Essays 1954-1979, Berlin, Weimar, AufbauVerlag, 1984, p. 80-116, ici p. 108.

13. Ibid., p. 109.

14. Ludwig Renn : «Weshalb keine Literatur über den Krieg? », op.cit., p. 126 sq.

15. Ibid., p. 127.

16. Cf. Ursula Heukenkamp : «Friedenskampf gegen Pazifismus. Die Konventionalisierung des Kriegsbildes nach 1945 », in Id. (dir.) : Militärische und zivile Mentalität. Ein literaturkritischer Report, Berlin, Aufbau-Taschenbuchverlag, 1991, p. 134-154, ici p. 148.

17. Article du Neues Deutschland du 8 janvier 1956 cité par U. Heukenkamp, ibid., p. 151.

18. Ibid., p. 152.

19. Cf. Martina Langermann : «Diskussionen um die „harte Schreibweise“... », op. cit., p. 1420.

20. Ibid., p. 1421.

21. Franz Fühmann : Kameraden, Berlin, Aufbau-Verlag, 1955.

22. Cf. Franz Fühmann : « Mein Erstling » [1973], in Sinn und Form, 2/1989, p. 273-279.

23. Les deux nouvelles sont d'abord publiées dans Neue deutsche Literatur (3/1957) et Sonntag ( ${ }^{\circ} 18$ à 20/1957), avant de figurer dans le recueil Stürzende Schatten. Novellen, Berlin, Verlag der Nation, 1959 (avec des illustrations de Hans et Lea Grundig).

24. Cette lecture est déjà suggérée par Dennis Tate : Franz Fühmann. Innovation and Authenticity. A study of his prose-writing, Amsterdam, Atlanta, Rodopi, 1997, p. 49.

25. Franz Fühmann : « Mein Erstling », op. cit., p. 279.

26. Ibid., p. 276.

27. Tous les récits analysés ici sont cités d'après l'édition suivante : Franz Fühmann : Erzählungen 1955-1975, Rostock, Hinstorff Verlag, 1982: Kameraden (p.7-48), Das Gottesgericht (p. 49-71), Kapitulation (p. 73-99) et Das Erinnern (p.101-118). Les numéros de page figurent entre parenthèses dans le texte.

28. Franz Fühmann : « Mein Erstling », op. cit., p. 276sq. 
29. Cf. Uwe Wittstock : Über die Fähigkeit zu trauern. Das Bild der Wandlung im Prosawerk von Christa Wolf und Franz Fühmann, Francfort/Main, Athenäum, 1987, p. 24 et 34sq.

30. Dennis Tate souligne que Kameraden est une des premières œuvres d'un auteur de RDA à défier une notion fondamentale du réalisme socialiste, celle d'être idéologiquement explicite. $\mathrm{Cf}$. Dennis Tate : Franz Fühmann. Innovation and Authenticity..., op. cit., p. 39.

31. Voir la lecture extrêmement simpliste d'Ingrid Dinter : Unvollendete Trauerarbeit in der DDRLiteratur. Ein Studium der Vergangenheitsbewältigung, New York, Washington et al., Peter Lang, 1994, p. 87.

32. Contrairement à la lecture de Werner Baum qui croit en un possible sauvetage et suggère que Thomas peut commencer sa renaissance intellectuelle et morale chez les hommes soviétiques. Cf. Werner Baum: "Bedeutung und Gehalt». Über die sozialistische Novelle, Halle, Mitteldeutscher Verlag, 1968, p. 17-38, ici p. 36.

33. Dans Stürzende Schatten, Fühmann mentionne le titre allemand du récit de Bierce : «Es gibt kein Entrinnen » qui figure dans le recueil Erzähler von drüben (Amerikaner, Engländer, Franzosen), édité par Hans B. Wagenseil au Limes-Verlag Wiesbaden, Verlag des Druckhauses Tempelhof Berlin, 1947, p. 8-14.

34. Rosemarie Heise : «Die Bürde der Vergangenheit", in Neue deutsche Literatur 8/1959, p. 132-134, ici p. 133.

35. Certains parlent d'une « conversion comme catastrophe, comme conscience subite et tardive de sa propre culpabilité », mais ce constat abuse de la notion de «conversion » telle que l'utilise Fühmann. Cf. Heinrich Küntzel : «Der Faschismus : seine Theorie, seine Darstellung in der Literatur », in Hans-Jürgen Schmitt (dir.) : Die Literatur der DDR (Hansers Sozialgeschichte der deutschen Literatur, tome 11), München, Wien, Carl Hanser Verlag, 1983, p. 435-467, ici p. 454.

36. Cf. Stephan Hermlin : «Franz Fühmann», inHorst Simon (dir.) : Zwischen Erzählen und Schweigen. Ein Buch des Erinnerns und Gedenkens. Franz Fühmann zum 65., Rostock, Hinstorff Verlag, 1987, p. 45-40, ici p. 46.

37. Hans Richter : Franz Fühmann - Ein deutsches Dichterleben, Berlin, Weimar, Aufbau-Verlag, 1992, p. 142.

38. Cf. Jörg Echternkamp : «Arbeit am Mythos. Soldatengenerationen der Wehrmacht im Urteil der west- und ostdeutschen Nachkriegsgesellschaft ", in Klaus Naumann (dir.), Nachkrieg in Deutschland, op. cit., p. 421-443.

39. Ibid., p. 433.

40. Cf. Bill Niven : Facing the Nazi Past. United Germany and the Legacy of the Third Reich, Routledge, London and New York, 2002, p. 150.

41. Cf. Jürgen Danyel: "Die SED und die 'kleinen PG's'. Zur politischen Integration der ehemaligen NSDAP-Mitglieder in der SBZ/DDR ", in Annette Leo et Peter Reif-Spirek (dir.) : Helden, Täter und Verräter. Studien zum DDR-Antifaschismus, Berlin, Metropol Verlag, 1999, p. 177-196, ici : p. 185. Avec cette politique d'intégration, le SED faisait de la concurrence au parti qui fut fondé pour récupérer les anciens membres du NSDAP, le NDPD, auquel appartenait aussi Fühmann qui y occupait une fonction importante.

42. Les «frrht, vrrdg, schkslhft, gtt, ndkmpf, rrch, sndg [...]» (104) ressemblent en effet étonnamment aux expérimentations d'Ernst Jandl, notamment dans son poème "schtzngrmm » dont les sons imitent par ailleurs le bruit d'une mitrailleuse. Le poème de Jandl date de 1957, la même année, quelques-uns de ses poèmes expérimentaux ont été publiés dans une revue (le recueil «Laut und Luise » où figure le poème ne fut publié qu'en 1966). Nous ne savons pas si Fühmann en a pu avoir connaissance, mais il était particulièrement attentif à la poésie concrète dont témoigne son journal de Hongrie (voir l'article sur Jandl dans ce même recueil).

43. Lettre de Franz Fühmann à Ludvík Kundera du $1^{\mathrm{er}}$ novembre 1957, in Franz Fühmann : Briefe 1950-1984. Eine Auswahl, éd. par Hans-Jürgen Schmitt, Rostock, Hinstorff Verlag, 1994, p. 19. 
44. Cf. Klaus-Peter Merta : «Uniformierung als Mittel der Politik», in Dieter Vorsteher (dir.) : Parteiauftrag: ein neues Deutschland. Bilder, Rituale und Symbole der frühen DDR, Deutsches Historisches Museum, Berlin, 1996, p. 175-186.

45. Terme de Klaus Briegleb repris par Stephan Braese: «Unmittelbar zum Krieg - Alfred Andersch und Franz Fühmann », in Klaus Naumann (dir.) : Nachkrieg in Deutschland, Hamburg, Hamburger Edition, 2001, p. 472-497, ici p. 474.

46. Franz Fühmann : « Antwort auf eine Umfrage », op. cit., p. 19.

47. Franz Fühmann : «Die Fahrt nach Stalingrad», in Id. : Fronten, Berlin, Aufbau-Verlag, 1960, p. 111-160, ici : p. 122. (Ce recueil regroupe également les trois nouvelles de guerre).

48. Cf. Anne Lagny: «Franz Fühmann: Das Judenauto (1962). La 'Leçon' d'une enfance allemande? », in Germanica, 15/1994, p. 83-101, ici p. 89.

\section{RÉSUMÉS}

L'article s'intéresse aux premiers récits de Franz Fühmann sur la Seconde Guerre mondiale et les situe dans le contexte du discours aussi bien littéraire que politique sur le passé et sur la guerre dans la RDA des années 1950.

Écrites strictement dans la perspective de soldats allemands et évoquant des situations de crimes de guerre, les trois principales nouvelles («Kameraden», «Das Gottesgericht» et «Kapitulation») participent à maintenir présente la question de la culpabilité personnelle des soldats impliqués dans les crimes commis par la Wehrmacht, à une époque où le discours officiel visait à la déculpabilisation, entraînant un oubli volontaire en faveur de l'intégration des soldats dans la société.

Trois autres courts récits (regroupés sou le titre « Das Erinnern ») sont écrits dans la perspective de la remémoration de la période du national-socialisme et témoignent du passage du passé vers le présent que l'auteur pense maintenant en termes de transmission. Si le discours officiel interfère dans ces récits, la thématisation d'une impossibilité de transmettre l'expérience de la guerre remet en question, à l'instar des nouvelles, le consensus antifasciste dans la société estallemande.

Der Artikel behandelt Franz Fühmanns erste Erzählungen über den Zweiten Weltkrieg und verortet diese im Kontext des literarischen und politischen Diskurses über Vergangenheit und Krieg in der DDR der 1950er Jahre.

Die drei wichtigsten Novellen („Kameraden“, „Das Gottesgericht“ et „Kapitulation“) wurden aus der ausschließlichen Perspektive deutscher Soldaten geschrieben und handeln von Situationen, in denen Kriegsverbrechen begangen wurden. Dadurch halten sie die Frage nach der individuellen Schuld von in Wehrmachtsverbrechen verwickelten Soldaten wach, und zwar zu einer Zeit, da der offizielle Diskurs dazu tendierte, die Soldaten zur besseren Integration in die Gesellschaft von Schuld frei zu sprechen, und so die Verbrechen der Vergangenheit willentlich dem Vergessen preisgab.

Drei weitere kurze Erzählungen (zusammengefasst unter dem Titel „Das Erinnern“) wurden aus der Perspektive der Erinnerung an die NS-Zeit geschrieben und zeugen von der Überführung der Vergangenheit in die Gegenwart, wobei der Autor jetzt die Frage der Vermittlung aufwirft. Obwohl diese Erzählungen Spuren des offiziellen Diskurses aufweisen, stellt die Thematisierung 
der unmöglichen Vermittlung von Kriegserfahrungen, ebenso wie in den Novellen über den Krieg, den antifaschistischen Konsens der ostdeutschen Gesellschaft in Frage.

INDEX

Mots-clés : guerre, RDA

\section{AUTEURS}

CAROLA HÄHNEL-MESNARD

École Polytechnique 